\title{
Changing the Risk Threshold for Surgical Prevention of Ovarian Cancer
}

${ }^{*}$ Ranjit Manchanda ${ }^{1,2,3}$, Rosa Legood ${ }^{4}$, Antonis C Antoniou ${ }^{5}$, Leigh Pearce ${ }^{6,7}$, Usha Menon $^{3}$

${ }^{1}$ Barts Cancer Institute, Queen Mary University of London, Old Anatomy Building, Charterhouse Square, London, EC1M 6BQ, UK

${ }^{2}$ Department of Gynaecological Oncology, St Bartholomew's Hospital, London, UK, EC1A 7BE ${ }^{3}$ Gynaecological Cancer Research Centre, Department of Women's Cancer, Institute for Women's Health, University College London, 149 Tottenham Court Road, London, UK, W1T 7DN.

${ }^{4}$ Department of Health Services Research and Policy, London School of Hygiene and Tropical Medicine, 15-17 Tavistock Place, London, WC1H 9SH

${ }^{5}$ Centre for Cancer Genetic Epidemiology, Department of Public Health and Primary Care, University of Cambridge, Strangeways Research Laboratory, Worts Causeway, Cambridge, CB1 8RN, UK.

${ }^{6}$ Department of Epidemiology, School of Public Health, University of Michigan, Ann Arbor, Michigan, 48109

${ }^{7}$ Department of Preventive Medicine, USC Keck School of Medicine, University of Southern California, Los Angeles, California 900

\section{*Correspondence to:}

Dr Ranjit Manchanda

Barts Cancer Institute, Queen Mary University of London,

Old Anatomy Building, Charterhouse Square, London, EC1M 6BQ, UK

Email: r.manchanda@qmul.ac.uk 


\section{Changing the Risk Threshold for Surgical Prevention of Ovarian Cancer}

Ovarian cancer $(\mathrm{OC})$ remains a lethal disease and a major cause of deaths from gynaecological cancer particularly in western countries. Despite advances in treatment, there have been only marginal improvements in survival rates over the last 30years. Additionally, the number of OC cases in the UK is estimated to increase by $27-45 \%$, which translates to a $15 \%$ rise in the incidence rate by 2035. ${ }^{1}$ A national OC screening programme is unavailable as this has not yet shown a conclusive mortality benefit. ${ }^{2}$ Hence, primary prevention continues to be the cornerstone in reducing the burden of $\mathrm{OC}$ and improving health outcomes. This is essential to achieve long term transformational change and cost-efficiencies in our health-system. Its importance is amplified by current economic restraints, with a $2.4 \%$ funding shortfall reported in the UK National-HealthService (NHS).

Risk-reducing salpingo-oophorectomy (RRSO) is the most effective method of preventing OC. It is usually feasible as a laparoscopic day-case procedure though an overnight stay may at times be needed. This option of surgical prevention has traditionally been offered to high-risk women with BRCA1/BRCA2 mutations or those with Lynch syndrome, in whom with lifetime OC-risk ranging from $10 \%$ to $40 \%$. The procedure has been shown to be cost-effective in this patient population. ${ }^{3}$ In BRCA1/BRCA2 carriers RRSO is associated with a 79\% reduction in OC-risk ( $\mathrm{HR}=0.21, \mathrm{Cl}: 0.12,0.39)^{4}$ and a reduction in $\mathrm{OC}$ and all-cause mortality. ${ }^{5}$ There is a small $2-4 \%$ residual risk of primary peritoneal cancer over 20years follow-up.

Amongst average risk women RRSO is associated with a 94\% reduced risk of OC ( $H R=0.06$, $\mathrm{Cl}: 0.02,0.17) .{ }^{6}$ RRSO could potentially be a cost-effective option for this group of women as well. This had not previously been explored. We recently estimated the OC-risk thresholds at which RRSO would be cost-effective in pre-menopausal ${ }^{7}$ and post-menopausal ${ }^{8}$ women. To do this we used a 
decision-analytic modelling framework. Our decision analytic modelling shows that pre-menopausal RRSO is cost-effective at $>4 \%$ lifetime $O C$ absolute risk with an incremental cost-effectiveness ratio $($ ICER $)=f 19536 /$ quality-adjusted life-year (QALY) leading to an averaged $>42.7$ days gain in lifeexpectancy, if hormone-replacement-therapy (HRT) compliance is high. ${ }^{7}$ In postmenopausal women $>50$ years, RRSO is cost-effective at $\geq 5 \%$ lifetime OC-risk levels with an ICER $=£ 15247 / \mathrm{QALY}$ and increases life-expectancy by an average $>29.2$ days. $^{8}$ Above these risk levels, RRSO saves more lifeyears and QALYs and is highly cost-effective at the National Institute for Care-\&-Health Excellence (NICE) $£ 20,000-30,000 / Q A L Y$ willingness-to-pay threshold. While the number of days gained in lifeexpectancy appear small, it is important to highlight these values are averaged across the population, and hence, for the individual woman in whom OC is prevented, this is many fold higher. As a comparator, undergoing cervical cancer screening leads to a $11.6-32.4$ days,${ }^{9}$ and colorectal cancer screening leads to a 55-91days average gain in life-expectancy. ${ }^{10}$ These analyses fulfil NICE requirements for health-economic decision making and are necessary for assessing the balance of costs and consequences for recommending policy changes related to new interventions, such as new thresholds for surgical prevention.

It is estimated that around $9 \%$ of the UK population has an OC-risk $\geq 5 \%$, and this group accounts for $53 \%$ of OC cases, while $13.4 \%$ of the population is at $>4 \%$ risk and $62.8 \%$ OC cases occur in them. ${ }^{11}$ The availability of data supporting surgical prevention at intermediate $5 \%-10 \%$ levels of OC-risk provides a significant opportunity to reduce OC incidence and make a much greater impact on burden of this fatal disease. Genetic testing for more recently identified moderate-risk OC genes RAD51C, RAD51D and BRIP1, is now clinically available. Mutations in these genes are associated with lifetime risks in the range $5.8 \%$ to $11 \%$, although confidence intervals are wide. As more data accumulate, the precision of these estimates will improve and Cls narrow. We feel RRSO should be made available to unaffected women with these moderate penetrance mutations who have completed their family after proper counselling. OC has not yet been reported under $40 y e a r s$ age in 
RAD51C and RAD51D carriers or under 50 years in BRIP1 carriers. Hence, surgical prevention is best delayed till $>40$ or $>50 y e a r s$ in these women respectively. First-degree-relatives of women with OC and BRCA1/BRCA2 negative women (without a known pathogenic mutation in the family) but with a strong family history of $\mathrm{OC}$ or $\mathrm{OC}$ 'and' breast cancer (BC) are also at intermediate-risk and could qualify for surgical prevention. In intermediate-risk women with no gene mutation we would suggest RRSO after 50 or the menopause.

We recently published OC-risk models which can identify women at these intermediate-risk thresholds. ${ }^{11,12}$ Incorporating SNPs/common genetic-variants into models permit more accurate risk prediction. Three quarters of the familial relative risk for $O C$ is not accounted for by $B R C A$ mutations and using a SNP based polygenic-risk-score in combination with family-history can identify women above the $5 \%$ risk threshold and improve risk prediction in BRCA negative women. ${ }^{11}$ Using a combination of known OC risk factors of endometriosis, tubal ligation, parity, contraceptive pill, and family history (first-degree-relative with OC) along with SNP profile, it is possible to identify eight categories with a $\geq 5 \%$ life time OC-risk. ${ }^{12}$ The authors estimated that $2 \%$ of women in the USA would be at $\geq 5 \%$ lifetime OC-risk. ${ }^{12}$

More sophisticated models incorporating epidemiological data, next generation sequencing driven panel-testing, moderate penetrance genes, and increasing number of OC SNPs are under development within the PROMISE (Predicting-Risk of Ovarian-Malignancy Improved-Screening \& Early-detection) programme and OCAC (Ovarian Cancer Action Consortium). As these models get validated and made available, the capability to predict risk will increase and its clinical application will expand even more in the near future. Going forward, it could provide a potential opportunity for population-based risk stratification for OC for the purpose of targeted surgical prevention. A pilot study to assess feasibility of such an approach will commence in summer of 2017. 
Limitations:

While acceptability of RRSO for high penetrance gene mutations is well established with uptake rates of up to $70 \%$ reported, the acceptability and uptake at intermediate-risk thresholds is unknown as RRSO was not previously available at these levels. The risk of surgical complications from RRSO is around 3\% and needs to be an important part of informed decision making on whether to undergo the procedure. Another important issue for women undergoing premenopausal oophorectomy is the detrimental consequences of premature surgical menopause. It is associated with osteoporosis, vasomotor symptoms, increased risk of cardiovascular-disease, and detrimental impact on sexualfunction. An absolute increase in cardiovascular mortality of $3.03 \%$ has been described in women who do not take HRT. ${ }^{6}$ The harmful side effects can be ameliorated by HRT and are seen predominantly in women $<45$ years who decline or are unable to have HRT. Studies suggest that some of the protective neurocognitive effects may not however be fully addressed by HRT. Short term HRT given till the age of 50years following premature surgical menopause has not been shown increase BC-risk. The importance of these issues in decision making is well documented in high-risk women, such as BRCA1/BRCA2 carriers as a number of them delay RRSO till after menopause. Despite the downsides, high satisfaction rates of $>85 \%$ are reported with RRSO in BRCA1/BRCA2 carriers. Corresponding data for intermediate-risk women is not available.

Role of Hysterectomy:

The utility of concomitant hysterectomy as part of the surgical procedure with RRSO to prevent OC has been debated. Practice varies in different parts of the world, with hysterectomy more prevalent in some countries like USA, Australia. In a number of these countries, a significant proportion of medical practice is private and insurance driven which may also influence differences in approach. Proponents of a concurrent hysterectomy cite the benefits of estrogen alone HRT and avoiding cervical smears. Hysterectomy is a major operation with significantly higher morbidity, longer operating time, hospital stay and recovery than bilateral salpingo-oophorectomy. We and most UK 
centres don't routinely undertake hysterectomy for OC prevention in high-risk women, for a number of reasons. Hysterectomy is not routinely offered as an alternate to progesterone component of HRT in gynaecological practice. The large number of women on HRT attending routine gynaecological menopause clinics are not offered this. Short-term HRT given in this context till the average age of menopause (51years) does not increase BC-risk. ${ }^{13}$ Mirena-IUS (intra-uterine-progestogen coil) with minimal systemic absorption can be used for the progestogenic component of HRT. While one recent report suggests the relative-risk of 'serous' endometrial-cancer (EC) may be increased in BRCA1 women, ${ }^{14}$ corroborating studies are awaited. Additionally serous-subtype comprises a very small proportion ( $6.5 \%)$ of ECs and the overall EC-risk is not significantly increased in BRCA1 women. Hence, it's not currently our practice to routinely offer this. Premenopausal $B R C A /$ high-risk women with ER+ BC on Tamoxifen, have a 2-3 fold increased risk of endometrioid EC. However, the absolute EC-risk is still very low (2.3cases/1000 women/year-of-use) ${ }^{15}$ with a favourable risk profile in pre-menopausal women and increased EC-risk reported in women $\geq 50$ years. ${ }^{15}$ We do not feel this risk justifies routine hysterectomy but any unscheduled/abnormal bleeding promptly investigated. This is consistent with ACOG and RCOG guidelines on Tamoxifen. One clear exception is LynchSyndrome women (lifetime EC-risk $\sim 40 \%$ ), in whom hysterectomy is undertaken for surgical prevention.

Salpingectomy in high-risk women:

The increasing acceptance of the tubal origin of OC has led to premenopausal Early-Salpingectomy (ES) followed by delayed-oophorectomy (DO) at menopause, proposed as a two-step OC prevention strategy for high-risk women. This enables women to obtain some OC-risk reduction while averting detrimental consequences of premature menopause. However, prospective data are lacking for (a) long-term endocrine function and menopause onset, (b) precise level of OC-risk reduction obtained, (c) quality-of-life, (d) psychosocial impact. Concerns have also been expressed regarding long-term follow-up and attrition from DO. Some of them may develop OC and this procedure will have been a 
failure. ${ }^{16}$ While $70 \%$ occult insitu/invasive lesions identified at RRSO in BRCA1/BRCA2 women are tubal $30 \%$ are not. ${ }^{17}$ Our understanding of the roles and interaction of the tube-\&-ovary in OC etiopathogenesis is still incomplete and evolving. Not all STIC (serous-tubal-intraepithelialcarcinomas) are precursors of OC and some may be metastatic. ${ }^{18}$ Only up to $61 \%$ OCs have STICs associated with them. Salpingectomy will therefore not prevent all OC. Surgico-pathological protocols for undertaking ES and managing occult lesions need standardisation and utility-scores required for health-economic analysis need developing. Given limitations highlighted above, their remains significant uncertainty around the cost-effectiveness of an ES-strategy. It is best that ES-\&DO is evaluated/undertaken within the safe environment of a clinical trial not routine practice. Salpingectomy studies are underway in France (Radical-Fimbriectomy-study NCT01608074), USA (MD Anderson: NCT01907789), The Netherlands (TUBA-study NCT02321228). A UK-study (PROTECTOR) will commence later this year.

The contraceptive pill:

There is good evidence to show that oral contraceptive pill (OCP) use significantly reduces OC-risk in average-risk women. ${ }^{19}$ The level of risk-reduction increases with duration of use and, benefits persist for 30 years after stopping the pill. The risk-reduction obtained is $22 \%$ with $1-4$ years use, $35 \%$ after $5-9$ years use, $45 \%$ with $10-14$ years and $58 \%$ with $\geq 15$ years use. ${ }^{19}$ Data from high-risk populations suggest similar benefits are found in BRCA1/BRCA2 carriers, with 5-years' OCP use halving the OCrisk (risk-reduction ranges from $33 \%-80 \%$ in $B R C A 1,58 \%-63 \%$ in $B R C A 2) .{ }^{20}$ Although specific data for intermediate-risk women is lacking, OCP-use is likely to provide similar benefits to them too. A disadvantage is an increased $\mathrm{BC}$-risk in average-risk women ( $\mathrm{RR}=1.24, \mathrm{Cl}: 1.15,1.33$ ) and for up to 10years after stopping the pill. However, the absolute increase in BC-risk is small. OCP is contraindicated in women who have had BC but not in those with a family-history of BC. Studies evaluating the impact of OCP on $\mathrm{BC}$-risk in BRCA1/BRCA2 carriers show conflicting results. Metaanalysis from case-control data show no increase in BC-risk (BRCA1 ES=0.78, Cl:0.59-1.04; BRCA2 
$\mathrm{ES}=1.04, \mathrm{Cl}: 0.81-1.32),{ }^{20}$ but, cohort data report an increased $\mathrm{BC}$-risk (BRCA1 ES=1.59, Cl:1.32-1.92;

BRCA2 ES=1.85, Cl:1.30-2.64). ${ }^{20}$ The absolute increase in risk is probably small if taken at a young age. Progesterone-only-pill, implants and injectable contraception may be safer options.

The OC-risk threshold for surgical prevention has previously been set too high. We call for a review of this threshold. Changing this to around the $5 \%$ life-time risk level provides an important costeffective strategy for OC prevention. Widening access to RRSO will also require the need for health professionals to ensure that women who undergo premenopausal oophorectomy appreciate the importance and need for HRT till the age of natural menopause. A mechanism of ensuring compliance which includes sustained access to prescriptions and long term monitoring will be needed. Health service capacity issues including expansion in downstream management pathways will need to be addressed. The rising costs of OC drugs and treatment, persistent poor survival outcomes, lack of effective screening programme and predicted increase in burden of disease, emphasise the need for shifting focus towards better targeted cost-effective prevention. It is important we seize this opportunity afforded to maximise prevention of this devastating disease.

\section{Disclosure of Interests}

UM has a financial interest in Abcodia, Ltd, a company formed to develop academic and commercial development of biomarkers for screening and risk prediction. UM reports research funding from CRUK, MRC, NIHR and The Eve Appeal. RM reports research funding from CRUK, The Eve Appeal and The Barts and the London Charity into population based genetic testing, risk stratification and ovarian cancer prevention. The other authors declare no conflict of interest.

\section{Ethical Approval}

This commentary is not new research and did not require any ethical approval 


\section{Contribution to authorship}

RM prepared and initial draft which was critically contributed to by all authors $U M, A A, L P, R L$. All authors approved final version of the manuscript.

\section{Role of Funding Source}

The manuscript is not funded by any charity or grant. 


\section{References}

1. International Agency for Research on Cancer. GLOBOCAN 2012: Estimated Cancer Incidence, Mortality and Prevalence Worldwide in 2012. Online Analysis > Prediction. Lyon, France: IARC (International Agency for Research on Cancer); 2016. p.

http://globocan.iarc.fr/Pages/burden sel.aspx.

2. Jacobs IJ, Menon U, Ryan A, Gentry-Maharaj A, Burnell M, Kalsi JK, et al. Ovarian cancer screening and mortality in the UK Collaborative Trial of Ovarian Cancer Screening (UKCTOCS): a randomised controlled trial. Lancet. 2015 Dec 16.

3. Anderson K, Jacobson JS, Heitjan DF, Zivin JG, Hershman D, Neugut Al, et al. Costeffectiveness of preventive strategies for women with a BRCA1 or a BRCA2 mutation. Ann Intern Med. 2006 Mar 21;144(6):397-406.

4. Rebbeck TR, Kauff ND, Domchek SM. Meta-analysis of risk reduction estimates associated with risk-reducing salpingo-oophorectomy in BRCA1 or BRCA2 mutation carriers. J Natl Cancer Inst. $2009 \operatorname{Jan} 21 ; 101(2): 80-7$.

5. Domchek SM, Friebel TM, Singer CF, Evans DG, Lynch HT, Isaacs C, et al. Association of riskreducing surgery in BRCA1 or BRCA2 mutation carriers with cancer risk and mortality. JAMA. 2010 Sep 01;304(9):967-75.

6. Parker WH, Feskanich D, Broder MS, Chang E, Shoupe D, Farquhar CM, et al. Long-term mortality associated with oophorectomy compared with ovarian conservation in the nurses' health study. Obstet Gynecol. 2013 Apr;121(4):709-16.

7. Manchanda R, Legood R, Antoniou AC, Gordeev VS, Menon U. Specifying the ovarian cancer risk threshold of 'premenopausal risk-reducing salpingo-oophorectomy' for ovarian cancer prevention: a cost-effectiveness analysis. J Med Genet. 2016 Sep;53(9):591-9. 
8. Manchanda R, Legood R, Pearce L, Menon U. Defining the risk threshold for risk reducing salpingo-oophorectomy for ovarian cancer prevention in low risk postmenopausal women. Gynecol Oncol. 2015 Oct 5.

9. van den Akker-van Marle ME, van Ballegooijen M, van Oortmarssen GJ, Boer R, Habbema JD. Cost-effectiveness of cervical cancer screening: comparison of screening policies. J Natl Cancer Inst. 2002 Feb 6;94(3):193-204.

10. Knudsen AB, Zauber AG, Rutter CM, Naber SK, Doria-Rose VP, Pabiniak C, et al. Estimation of Benefits, Burden, and Harms of Colorectal Cancer Screening Strategies: Modeling Study for the US Preventive Services Task Force. JAMA. 2016 Jun 21;315(23):2595-609.

11. Jervis $\mathrm{S}$, Song $\mathrm{H}$, Lee $\mathrm{A}$, Dicks $\mathrm{E}$, Harrington $\mathrm{P}$, Baynes $\mathrm{C}$, et al. A risk prediction algorithm for ovarian cancer incorporating BRCA1, BRCA2, common alleles and other familial effects. J Med Genet. 2015 May 29.

12. Pearce CL, Stram DO, Ness RB, Stram DA, Roman LD, Templeman C, et al. Population distribution of lifetime risk of ovarian cancer in the United States. Cancer Epidemiol Biomarkers Prev. 2015 Apr;24(4):671-6.

13. Rebbeck TR, Friebel T, Wagner T, Lynch HT, Garber JE, Daly MB, et al. Effect of short-term hormone replacement therapy on breast cancer risk reduction after bilateral prophylactic oophorectomy in BRCA1 and BRCA2 mutation carriers: the PROSE Study Group. J Clin Oncol. 2005 Nov 1;23(31):7804-10.

14. Shu CA, Pike MC, Jotwani AR, Friebel TM, Soslow RA, Levine DA, et al. Uterine Cancer After Risk-Reducing Salpingo-oophorectomy Without Hysterectomy in Women With BRCA Mutations. JAMA oncology. 2016 Nov 01;2(11):1434-40.

15. Fisher B, Costantino JP, Wickerham DL, Cecchini RS, Cronin WM, Robidoux A, et al. Tamoxifen for the prevention of breast cancer: current status of the National Surgical Adjuvant Breast and Bowel Project P-1 study. J Natl Cancer Inst. 2005 Nov 16;97(22):1652-62. 
16. Chandrasekaran D, Menon U, Evans G, Crawford R, Saridogan E, Jacobs C, et al. Risk reducing salpingectomy and delayed oophorectomy in high risk women: views of cancer geneticists, genetic counsellors and gynaecological oncologists in the UK. Fam Cancer. 2015 Dec;14(4):521-30.

17. Powell CB. Risk reducing salpingo-oophorectomy for BRCA mutation carriers: twenty years later. Gynecol Oncol. 2014 Feb;132(2):261-3.

18. Eckert MA, Pan S, Hernandez KM, Loth RM, Andrade J, Volchenboum SL, et al. Genomics of Ovarian Cancer Progression Reveals Diverse Metastatic Trajectories Including Intraepithelial Metastasis to the Fallopian Tube. Cancer Discov. 2016 Dec;6(12):1342-51.

19. Collaborative Group on Epidemiological Studies of Ovarian C, Beral V, Doll R, Hermon C, Peto R, Reeves G. Ovarian cancer and oral contraceptives: collaborative reanalysis of data from 45 epidemiological studies including 23,257 women with ovarian cancer and 87,303 controls. Lancet. $2008 \operatorname{Jan} 26 ; 371(9609): 303-14$.

20. Friebel TM, Domchek SM, Rebbeck TR. Modifiers of cancer risk in BRCA1 and BRCA2 mutation carriers: systematic review and meta-analysis. J Natl Cancer Inst. 2014 Jun;106(6):dju091. 